\title{
PROPUESTA METODOLÓGICA COOPARTICIPATIVA PARA FORMULAR POLÍTICAS PÚBLICAS EN EL MARCO DEL PLAN REGIONAL CONCERTADO EN SALUD Y LOS DERECHOS HUMANOS*
}

\author{
Scarsi Maratuech, Rossana ${ }^{1}$, Casallo Véliz, Soledad, Pérez Gutarra, Guillermina y Habich Scarsi, Augusto G. \\ Facultad de Enfermería de la Universidad Nacional del Centro del Perú
}

\begin{abstract}
RESUMEN
Nuestro país y por ende nuestra región han tratado de encontrar un norte que oriente su desarrollo integral, los indicadores reflejan que los intentos parecen haber sido frustrantes, probablemente por que no se tomaron en cuenta los aportes de los diferentes actores sociales y marginaron a la sociedad. Las nuevas formas de lograr el desarrollo necesita de soluciones innovadoras para acabar con la problemática sociosanitaria y que influir en políticas públicas debe ser parte de los esfuerzos de los decisores para lograr cambios duraderos En este contexto, la investigación tiene como objetivo general: Determinar una propuesta metodológica cooparticipativa según opinión de los decisores para formular Políticas Públicas en Salud. Es una investigación social aplicativa de corte cuantitativo y cualitativo. Se utilizó el método exploratorio descriptivo analítico, las técnicas de recolección de datos fueron la encuesta y entrevista semiestructurada. La muestra estuvo constituida por 8 autoridades de la Dirección Regional de Salud y 2 de Gobierno Regional - Junín. Se tuvo como resultado principal que la metodología cooparticipativa para la generación de Políticas Públicas en Salud que sería efectiva para nuestra realidad, según opinión del $80 \%$ de la muestra, debe incluir: Identificación del problema, personas y organizaciones que participan en la política, Proceso de formulación, asignación de recursos y planes, redacción de la política enmarcada en un determinado contexto político, social, cultural y económico. La propuesta es cercana a propuestas planteadas por diferentes autores, sin embargo no consideran el proceso de implementación a través de programas y proyectos, pudiendo ser una gran falencia para el logro de objetivos. El estudio evidencia grados de desencuentro entre los campos de la salud y la generación de propuestas políticas relacionados con el débil ejercicio de rectoría, la fragmentación y gobernabilidad. Contiene rutas tendientes a establecer un enfoque interdisciplinario en el ámbito de la salud y fortalecer el diálogo entre los diversos actores sociales.
\end{abstract}

Palabras clave: Derechos Humanos, Políticas Públicas, Desarrollo Integral

\section{COOPARTICIPATIVE IN METHODOLOGICAL PROPOSAL FOR FORMULATING PUBLIC POLICIES IN THE THEME OF REGIONAL PLANS ACCORDING TO HEALTH AND HUMAN RIGHTS}

\begin{abstract}
Our country and there fore our region have always tried to find a direction (north) that leads its integral development, the indicators reflect that the attempts seem to have been frustrating probably because they didn't take into account the different social actors contribuitions marginating the society. The new ways to get such development need innovating solutions to end the socios sanilary problematic and the influence in public policy must be part of the effort of the leaders (decisors) to obtain great changes in this context. The investigation has as a main goal to determine a coopartacipative methodological proposal according to the opinion of the decisors to formulate Public policies health. It is a social applied investigation both quantitative and qualitative. We used exploratory, descriptive, audition method; the techniques of Data collection were the survey and semi structural interview. The sampled was conformed by 8 representative authorities of regional health directions and 2 Govern Regional representatives - Junin. It was gotten as main results that... the coo participative methodology for the generation of public policies in health would be effective for our reality. According to the opinion from $80 \%$ of the sample, it should include, the identification of problems persons and organizations that participate in this policy process of formulation allocation of resources and plans, editing of the policy in a determined socio cultural political and economical context. The proposal is similar to other proposals set for different authors, however they don't consider the process of implementation through a programme and projects, which could be a dificultin achieving the goals.
\end{abstract}

*. Este trabajo de investigación fue recibido el 20/12/2006 retornado para su revisión 20/06/2007 y aprobado para su publicación 10/12/2007

1. Email: roscarsi76@yahoo.es 
The studies demonstrates degrees of mixing Ups between areas in health and the generation of proposal policies related is a weak leadership of sector, the fragmentation and governability. It contains routes to establish an interdisciplinary approach in the health are and the streaghenicy a dialog among the different social actors

Key words: Human rigts, publicy, integral development.

\section{INTRODUCCIÓN}

La descentralización que es una política muy bien acogida luego de los antecedentes que el país tiene en materia de un exacerbado centralismo demanda cada vez más la formulación e implementación de políticas públicas como nuevas formas de lograr el desarrollo reconociendo que se necesitan soluciones innovadoras para acabar con la problemática sociosanitaria, y que influir sobre ellas debe ser parte de los esfuerzos para lograr cambios duraderos, hecho que obliga a cada gobernante a buscar formas de redistribuir funciones y propiciar la participación ciudadana en el marco de los derechos fundamentales para tener ventajas competitivas y obtener resultados sin sufrir costos políticos por un mal desempeño.

En este contexto, el presente trabajo titulado "Propuesta Metodológica Cooparticipativa para formular Políticas Públicas en el marco del Plan Regional Concertado en Salud y los Derechos Humanos" pretende ser un aporte para todos aquellos que consideran que las políticas públicas son importantes para direccionar un desarrollo sostenido y sustentable. El trabajo recoge opiniones de autoridades regionales que pueden permitir mejorar la agenda social y los programas de gobierno en el marco de la descentralización.

El objetivo del trabajo fue: determinar una propuesta metodológica cooparticipativa según opinión de los decisores para formular Políticas Públicas en el marco del Plan Regional Concertado en Salud, y los Derechos Humanos.

Infiriendo que las Políticas Públicas en Salud enmarcan un proceso compuesto de pasos y metodologías con grados de libertad y que todavía son entendidas en forma superficial por algunos de los funcionarios de las entidades afines.

\section{MATERIAL Y MÉTODOS}

Por la naturaleza del estudio se utilizó el método exploratorio descriptivo analítico, por cuanto en la región no existen investigaciones en el área. Es una investigación social de corte-cuantitativo y cualitativo.

Los métodos de investigación que se utilizaron fueron el método básico de la ciencia, además del inductivo, deductivo, estadístico y analítico.

Se tuvo en consideración las actividades preliminares, las del campo y de gabinete.
La población estuvo constituida por autoridades a nivel de la Dirección Regional de Salud y Gobierno Regional de Junín. La muestra fue de 10 autoridades: 8 de la Dirección Regional de Salud Junín y 2 del Gobierno Regional. Se tuvo en cuenta como criterio de inclusión la función de toma de decisiones en la formulación, implementación y evaluación de políticas públicas.

Para la recolección de información, en un primer momento se utilizó como técnica la encuesta siendo el instrumento el cuestionario, el cual estuvo orientado a obtener información sobre la metodología utilizada y/o conocida para la formulación de políticas públicas, en relación a una propuesta metodológica cooparticipativa para formular políticas públicas en el marco del Plan Regional Concertado en Salud y los Derechos Humanos. En un segundo momento se utilizó la entrevista personal para recabar información sobre la experiencia de las autoridades sobre formulación de políticas públicas.

\section{RESULTADOS}

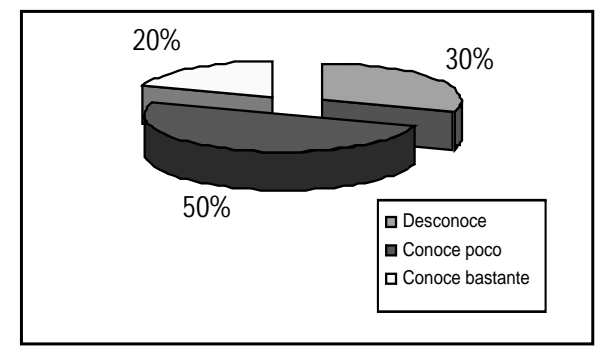

Gráfico 1. Conocimiento sobre Políticas Públicas en el Sector Salud.

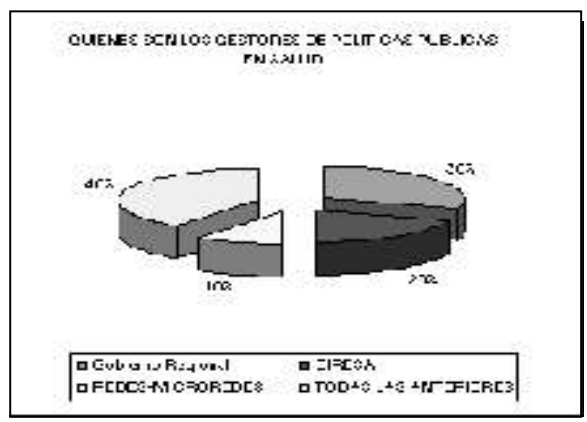

Gráfico 2. Gestores de polítcas públicas en salud. 


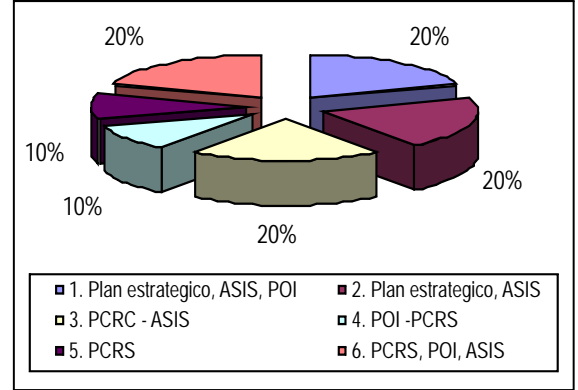

Gráfico 3. Los actores claves tiene informacion sobre políticas públicas, opinión de los encuestados sobre las fuentes que proporcionan información relevante para la identificación de problemas que amediten una política pública.

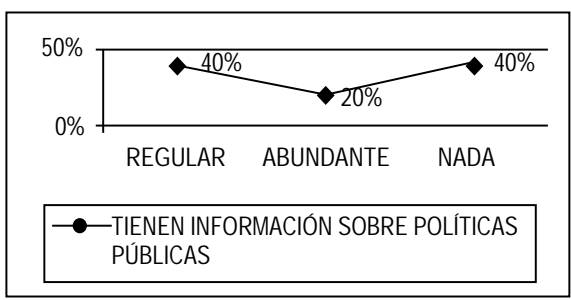

Gráfico 4. Tiene información sobre políticas públicas.

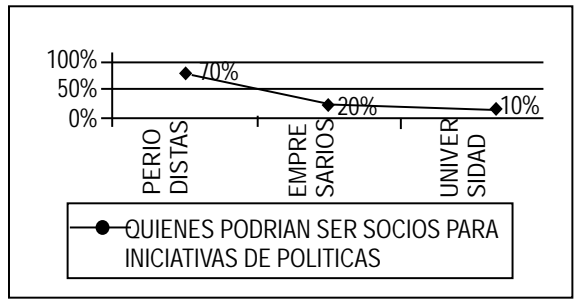

Gráfico 5. Quienes podrían ser socios para iniciativas de politicas públicas

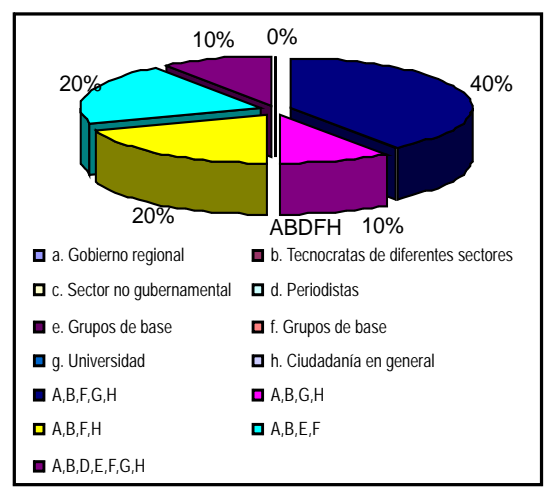

Gráfico 6. Opinión de los encuestados sobre los actores e instituciones que deben participar en la formulación de políticas públicas.

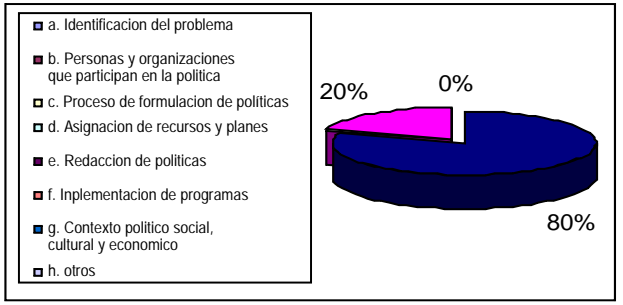

Gráfico 7. Según opinión de los encuestados, la propuestas metodología cooparticipativa efectiva para la formulación de políticas publicas en el marco del plan regional concertado de salud.

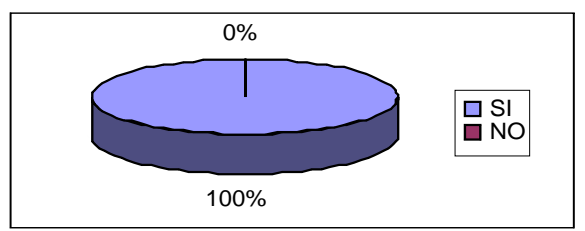

Gráfico 8. Según opinión de los encuestados el Plan Regional concertado en salud incluye políticas públicas según política de los encuestados.

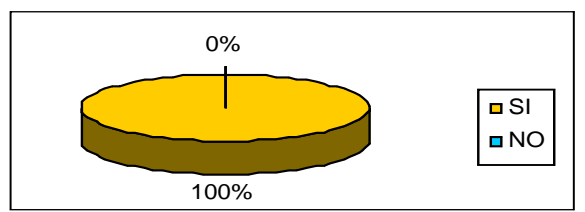

Gráfico 9. Según opinión de los encuestados el plan regional concertado en salud incluye el enfoque de derechos humanos.

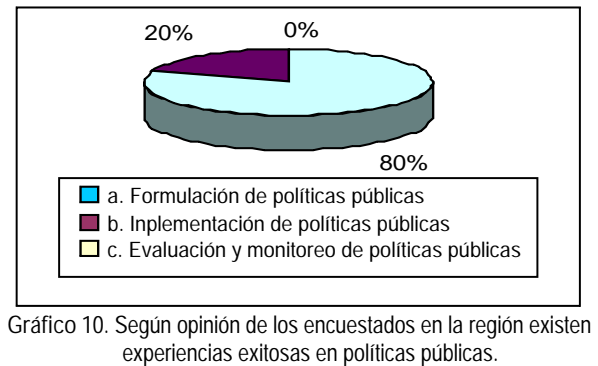

La política pública en salud enmarca las reglas y mecanismos que permiten el ejercicio o cambio relacionado a la concentración y cambio de poder, La complejidad del campo de las políticas públicas es fruto de los diferentes y muchas veces contradictorios factores que determinan su configuración y dinámica.

En este contexto podemos inferir que las políticas públicas en salud aunque sean delimitadas por las acciones públicas gubernamentales o no; están volcadas para dar respuesta a la demanda que tiene origen en el proceso de reproducción de los individuos en una sociedad, su complejidad involucra una dimensión valorativa fundada en un consenso social y que permiten escalonar prioridades y tomar decisiones. 
La materialización del ejercicio de los derechos humanos en el campo de las políticas públicas como en otros aspectos, no suele ser sencilla. Si bien su reconocimiento en nuestra región es un avance importante y es el resultado de un arduo trabajo de los actores involucrados en el área, su aplicación efectiva es bastante compleja.

La formulación de políticas públicas en salud, según evidencias encontradas es un tema que se requiere fortalecer en nuestra región. En relación a la propuesta metodológica cooparticipativa acorde a nuestra realidad para la formulación de políticas públicas en salud el $80 \%$ de decisores (autoridades encuestadas) tiene una propuesta muy cercana a las propuestas planteadas por diferentes autores, sin embargo no se considera en forma muy relevante el proceso implementación de las mismas a través de programas y proyectos pudiendo ser ésta una gran falencia para el logro de objetivos.

La formulación de políticas ocurre en espacios ampliamente variantes en aspectos económicos, sociales, culturales y niveles de desarrollo. Sin embargo, para su implementación es necesario la voluntad política; para lograr la sostenibilidad.

Es importante reconocer que en nuestra región se han dado grandes avances para la formulación de políticas, sin embargo todavía es débil su implementación y no existen mecanismos claros de sostenibilidad.

Se puede evidenciar que se ha reconocido la importancia de la participación de los diferentes actores sociales, sobre todo de la ciudadanía, hecho que permite fortalecer los derechos humanos y la construcción de democracia en el marco del proceso de descentralización y reforma del Estado.

\section{CONCLUSIONES}

El $50 \%$ de los entrevistados refiere conocer poco sobre políticas públicas en salud, el 30\% desconoce, (gráfico 1) hecho que puede limitar el tomar acciones para solucionar problemas en forma compartida.

- En relación a quienes deben de ser gestores de las Políticas Públicas en Salud, el 30\% de entrevistados (gráfico 2) refieren que el Gobierno Regional, hecho que se enmarca en el proceso de descentralización y reforma del Estado, siendo una competencia de dicha institución remarcando el rol rector de la Dirección Regional de Salud. Sin embargo, se evidencia opiniones que enmarcan a organizaciones que tiene el rol de operativisarlas.

- El $70 \%$ de los entrevistados refiere que los socios; para generar iniciativas de políticas públicas en salud, serian los periodistas, hecho que se refuerza con experiencia exitosa en formulación, implementación y vigilancia de políticas, en la medida que son actores que permiten la construcción de agenda publica (advocacy) y sirven de puente de diálogo entre los decisores y la población (Gráfico 3).

- El $40 \%$ de los entrevistados refieren que no cuentan con información sobre la formulación de políticas públicas y otros afines, hecho que amerita un urgente fortalecimiento de capacidades, jugando la universidad un rol preponderante (Gráfico 4).

- Los entrevistados refieren que las fuentes básicas para la recolección de información que permita una adecuada formulación de problemas para la generación de políticas públicas en salud son: el Plan Regional Concertado en Salud, El Plan Estratégico Institucional, El Plan Operativo Institucional, el Análisis Situacional de Salud y las normas pertinentes (Gráfico 5).

- A opinión de los entrevistados, en el campo de la salud, crear un ambiente para solucionar problemas requiere de la intervención de diferentes actores sociales, considerados como socios para iniciativas de políticas, remarcando la participación de los periodistas como un medio para generar agenda pública y ser un puente de dialogo entre las autoridades y la ciudadanía (Gráfico 6).

- El Plan Regional Concertado en Salud (PRCS), contiene propuestas que pueden conllevar a la formulación de políticas públicas, hecho que ha sido reconocido y a opinión de los entrevistados se están generando estrategias para la generación de las mismas, considerando que es un documento flexible. Los derechos humanos son considerados como ejes principales para la implementación del PRCS (Gráficos 7 y).

- La metodología cooparticipativa para la generación de políticas públicas en el marco del Plan Regional Concertado en Salud y los derechos humanos que sería efectiva para nuestra realidad, según opinión del $80 \%$ de autoridades entrevistadas / encuestadas debe incluir: Identificación del problema, personas y organizaciones que participan en la política, Proceso de formulación de políticas, asignación de recursos y planes ,redacción de la política y todo ello enmarcado en un determinado contexto político, social, cultural y económico. La propuesta que plantean es muy cercana a las propuestas planteadas por diferentes autores, sin embargo no consideran el paso de implementación de las mismas a través de programas y proyectos pudiendo ser está una gran falencia para el logro de objetivos (Gráfico 9).

- En relación a experiencias exitosas en la región Junín el $100 \%$ de entrevistados / encuestados, refieren que se aprobó las políticas de Recursos Humanos en Salud las cuales se encuentran en proceso de implementación. 
Sugiriendo que a través de la Universidad se debe de generar indicadores de monitoreo y evaluación (Gráfico 10).

\section{AGRADECIMIENTOS}

En nombre del equipo de investigación nuestro más sincero agradecimiento a las autoridades de Gobierno Regional Junín, Dirección Regional de Salud por el apoyo brindado y a todos aquellos actores sociales que hicieron posible la culminación del trabajo de investigación, fortaleciendo la concepción de que, la investigación ofrece respuestas importantes y valiosas en los diferentes ámbitos, facilitando un abordaje más sistémico de la realidad y la conexión entre el saber hacer y el saber ser orientado hacia el desempeño y la calidad de uso .

\section{LITERATURA CITADA}

Acuerdo Nacional Políticas de Estado y Foro del Acuerdo Nacional Compromiso Político, Social y Económico de 4to plazo. 2004. (Concordado en la ruta del gobierno 2004-2006).
Antonio, P. L., 2003. Derechos Humanos, estado de derecho y constitución. Madrid: Editorial Tecnos

Hardee, K; Fenanil; Boezwinkle, J; Clark, B. 2004. El círculo de la política. documento de trabajo de Policy $\mathrm{N}^{\circ}$ 11.

Hardee, K; Fenanil; Boezwinkle, J; Clark, B. 2004. Política en salud. documento de trabajo de Policy.

Huamán, A. L., MINSA. 2003. La gestión sanitaria en el marco del modelo de atención integral, DGSP, DEGS.

MINSA, 2003. La salud integral: compromiso de todos. El modelo de atención integral de salud,

MINSA, 2001. Lineamientos de política sectorial para el periodo 2002-2012 y fundamentos para el plan estratégico sectorial del quinquenio 2001-2006.

Stephen Marks The Human Rights, 2000. Framework to Development: Five Approaches, FXB Working Papers Series $n . ? 6,12$ ), disponible en http://www.hsph.harvard.edu/ 